\title{
Cannabinoid 1 receptor mediation of spinal cord ischemic tolerance induced by limb remote ischemia preconditioning in rats
}

\author{
Binxiao Su, MD, ${ }^{*}$ Hailong Dong, MD, PhD, ${ }^{*}$ Rui Ma, MD, Xijing Zhang, MD, PhD, Qian Ding, MD, and \\ Lize Xiong, MD, PhD
}

Objective: The aim of this study was to examine the influence of endogenous cannabinoids on neuroprotection of the spinal cord afforded by limb remote ischemic preconditioning.

\begin{abstract}
Methods: In experiment 1 (RIPC group), 3 cycles of limb remote ischemic preconditioning within different episodes $(2,3$, or 5 minutes) were induced before spinal cord ischemia in rats $(\mathrm{N}=5, \mathrm{n}=8)$. In experiment 2 , animals were pretreated intravenously by the vehicles, cannabinoid 1 (AM251, $1 \mathrm{mg} / \mathrm{kg}$ ) or cannaboid 2 (AM630,1 mg/kg) receptor antagonist 15 minutes before remote ischemic preconditioning, or else they were subjected to a sham operation. Thirty minutes after the pretreatment, spinal cord ischemia was induced $(\mathrm{N}=8, \mathrm{n}=8)$. In experiment 3 , the arachidonylethanolamide and 2-arachidonoylglycerol contents in the spinal cord after remote ischemic preconditioning and spinal cord ischemia were detected in rats $(\mathrm{N}=2, \mathrm{n}=12)$. Spinal cord ischemia was induced by 12 minutes of thoracic aorta occlusion in rats. Neurologic function was assessed 24 and 48 hours after reperfusion. Histopathologic examination was performed and the number of normal neurons in anterior spinal cord were counted.
\end{abstract}

Results: In experiment 1, 3 cycles of limb remote ischemic preconditioning ( 3 minutes of ischemia/ 3 minutes of reperfusion) induced ischemic tolerance on the spinal cords of the rats. The RIPC group showed a significant reduction in motor deficit index $(P<.01)$ as well as an increase in the number of normal neurons $(P<.01)$. In experiment 2, the cannabinoid 1 receptor antagonist AM251 pretreatment abolished the protective effects of remote preconditioning. In experiment 3 , arachidonylethanolamide content in spinal cord was elevated by remote ischemic preconditioning in rats.

Conclusion: These results indicated that endogenous cannabinoids, through acting on cannabinoid 1 receptors, were involved in the neuroprotective phenomenon on spinal cords of limb remote ischemic preconditioning. (J Thorac Cardiovasc Surg 2009;138:1409-16)

Ischemia/reperfusion (I/R) injury is the most important cause of paraplegia after thoracic aorta and spine surgery. The incidence of paraplegia is $16 \%$ as reported in thoracoabdominal aneurysm repair surgery ${ }^{1}$ and $11.8 \%$ in posterior longitudinal ligament surgery. ${ }^{2}$ Although a number of studies have shown that the risk of postoperative paraplegia has decreased with the application of new techniques, preventing spinal cord ischemic injury is still a big concern in surgery of the thoracic aorta and spine.

In past decades, ischemic preconditioning (IPC) has been proved to minimize $\mathrm{I} / \mathrm{R}$ injury in many organs including the spinal cord in animal models. ${ }^{3,4}$ Unfortunately, the idea of

\footnotetext{
From the Department of Anesthesiology, Xijing Hospital, Fourth Military Medical University, Xi'an, Shaanxi, China.

This work was supported by the National Science Foundation for Distinguished Young Scholars (Grant 30725039 to L.X.) and National Natural Science Foundation of China (Grant 30300329 to H.D.) and National Natural Science Foundation of China (Grant 30772074 to X.Z.).

* B.S. and H.D. are co-first authors.

Received for publication Feb 10, 2009; revisions received June 18, 2009; accepted for publication July 5, 2009; available ahead of print Aug 26, 2009.

Address for reprints: Lize Xiong, MD, PhD, Department of Anesthesiology, Xijing Hospital, Fourth Military Medical University, Xi'an 710032, Shaanxi, China (E-mail: lxiong@fmmu.edu.cn or hldong6@hotmail.com).

0022-5223/\$36.00

Copyright (c) 2009 by The American Association for Thoracic Surgery

doi:10.1016/j.jtcvs.2009.07.014
}

exposure to brief periods of ischemia in the spinal cord before an anticipated spinal cord ischemic event does not appear to be clinically relevant. Our previous studies showed that some nonischemic preconditioning methods, such as hyperbaric oxygen and isoflurane preconditioning, induced tolerance to spinal cord ischemia in an animal model $.^{5-7} \mathrm{Re}-$ cent studies also found that the IPC-induced ischemic tolerance occurred not only within the same tissue, but also between different regions as well as different organs. This phenomenon was called remote ischemic preconditioning (RIPC). ${ }^{8}$ RIPC has greater potential for clinical application than classic IPC inasmuch as it can be performed in a nonvital organ, avoiding the high risk of preconditioning in the vital organ, such as the brain or the spinal cord. Because RIPC was a more amenable and less invasive approach than other methods, it was applied to clinical study first during coronary arterial surgery and yielded satisfactory results. ${ }^{9,10}$

Despite the promising results of the clinical application of RIPC, the mechanisms responsible for this phenomenon and its signaling pathways still remained elusive. Numerous studies have suggested that the endocannabinoids were implicated in the protective effects of IPC through cannabinoid (CB) receptor-dependent mechanisms. ${ }^{11}$ The pharmacologic modulation of the endocannabinoid system 


$$
\begin{aligned}
& \text { Abbreviations and Acronyms } \\
& \mathrm{AEA}=\text { arachidonylethanolamide } \\
& 2-\mathrm{AG}=2 \text {-arachidonoylglycerol } \\
& \mathrm{CB}=\text { cannabinoid } \\
& \mathrm{IPC}=\text { ischemic preconditioning } \\
& \mathrm{I} / \mathrm{R}=\text { ischemia/reperfusion } \\
& \mathrm{MDI}=\text { motor deficit index } \\
& \mathrm{PaCO}_{2}=\text { arterial carbon dioxide tension } \\
& \mathrm{PaO}_{2}=\text { arterial oxygen tension } \\
& \mathrm{RIPC}=\text { remote ischemic preconditioning }
\end{aligned}
$$

represented a promising strategy in various cardiovascular, inflammatory, and metabolic disorders. A recent study has demonstrated that endogenous $\mathrm{CBs}$, by acting on $\mathrm{CB} 2$ receptors, were involved in the cardioprotective phenomenon of RIPC, induced by mesenteric artery occlusion and reperfusion. ${ }^{12}$ However, whether the endocannabinoid system mediated the spinal cord ischemic tolerance induced by limb RIPC remains to be further investigated.

Therefore, the primary objectives of the present study were to determine (1) whether limb RIPC could induce spinal cord ischemic tolerance and (2) whether it was mediated by endocannabinoids and $\mathrm{CB}$ receptors.

\section{MATERIALS AND METHODS}

The study was approved by the Ethics Committee for Animal Experimentation and all procedures were conducted according to the Guidelines for Animal Experimentation of our institutes. All animals were neurologically intact before anesthesia and were studied at Xijing Hospital, Fourth Military Medical University (Xi'an, China).

\section{Animals}

All experiments were performed on male Sprague-Dawley rats (300$350 \mathrm{~g}$ ). Rats were housed in standard cages with corncob bedding. Animals had access to food and water ad libitum and were housed separately after surgery. A 12-hour light/dark cycle (lights on at 7:00 AM) was maintained.

\section{Limb RIPC and Spinal Cord Ischemia}

The right femoral artery was separated below the right groin ligament for later induction of femoral artery occlusion (RIPC). Spinal cord ischemia was induced by the previously described technique. ${ }^{13}$ In brief, anesthesia was induced with $4 \%$ isoflurane in oxygen and maintained with $1.5 \%$ to $2 \%$ isoflurane. Body temperature was maintained at $37^{\circ} \mathrm{C}$ using a homeothermic blanket system. Distal arterial blood pressure and heart rate were monitored by a tail artery catheter (Colin BP 508; Colin Corporation, Komaki City, Japan). The blood samples were collected for arterial oxygen tension $\left(\mathrm{PaO}_{2}\right)$, arterial carbon dioxide tension $\left(\mathrm{PaCO}_{2}\right), \mathrm{pH}$, and plasma glucose measurement (Rapidlab 1200; Bayer, Leverkusen, Germany) from the tail artery. The left carotid artery was cannulated with a 20 -gauge polytetrafluoroethylene catheter for blood withdrawal and proximal arterial blood pressure. The left jugular vein was cannulated for intravenous drug injections. Spinal cord ischemia was induced by passage of a $2 \mathrm{~F}$ Fogarty catheter (American V. Mueller, CV 1035; Baxter, Irvine, Calif) through the left femoral artery to the descending thoracic aorta so that the tip reached the level of the left subclavian artery (10.8-11.4 cm from the site of insertion). The intraaortic balloon catheter was inflated with $0.05 \mathrm{~mL}$ of saline, and occlusion of aortic blood flow was confirmed by an immediate and sustained drop in distal arterial blood pressure. Systemic hypotension $(45 \mathrm{~mm} \mathrm{Hg})$ was maintained during occlusion by a blood-collecting circuit $\left(37.5^{\circ} \mathrm{C}\right)$ connected to the carotid artery and positioned at a height of $60 \mathrm{~cm}(45 \mathrm{~mm} \mathrm{Hg})$. After ischemia, the balloon was deflated, and the blood was reinfused during a 60 -second period. After blood reinfusion, $4 \mathrm{mg}$ of protamine sulfate was administered subcutaneously. Stabilization of arterial blood pressure was then monitored for an additional 10 minutes, after which the arterial lines were removed and wounds were closed. Rats were then allowed to recover.

\section{Neurologic and Histopathologic Evaluations}

The animals were neurologically assessed at 24 and 48 hours after reperfusion by an observer who was unaware of the grouping. To evaluate the neuronal damage, the observer evaluated motor function deficits in the hind limbs according to the criteria as reported. ${ }^{14}$ Motor deficit scores were graded according to the following scale: (a) walking/use of hind limbs: $0=$ normal, $1=$ toes flat under the body when walking, $2=$ knuckle walking, $3=$ movement of the hind limbs but unable to walk, and $4=$ no movement, dragging hind limbs; (b) placing/stepping reflex: $0=$ normal, $1=$ weak, and $2=$ not stepping. Each motor deficit score was obtained by adding the scores for scales $a$ and $b$.

A histopathologic evaluation was performed in the spinal cord for 48 hours after reperfusion. Rats were deeply anesthetized with sodium pentobarbital $(60 \mathrm{mg} / \mathrm{kg})$. Transcardiac perfusion and fixation were performed with $200 \mathrm{~mL}$ of $0.9 \%$ saline solution followed by $250 \mathrm{~mL}$ of $4 \%$ paraformaldehyde in $0.1 \mathrm{~mol} / \mathrm{L}$ phosphate buffer. The spinal cord was removed and postfixed in the same fixative overnight. Three representative sections were taken from L4 to L6 segments and were stained with hematoxylin and eosin for histopathologic evaluation. Neuronal injury was evaluated at a magnification of $\times 200$ by an observer who was unaware of the grouping. The remaining normal neurons in the ischemic ventral spinal cord in each animal, judged by their morphologic appearance, were counted in 3 sections selected randomly from the L4 to L6 segment and then averaged. The numbers of normal neurons per section were compared among groups.

\section{Experimental Protocol}

The statistical significance of histopathologic outcome was considered to be the end point in experiment 1 and experiment 2 . The statistical significance of arachidonylethanolamide (AEA) or 2-arachidonoylglycerol (2-AG) content was considered to be the end point of experiment 3 . The power of inequality test is set as 0.8 (alpha $=.05)$. Referring to our preliminary study, we assumed a difference in normal motor neurons and AEA content in spinal cord of about 20/slice and $0.4 \mathrm{pmol} / \mathrm{mg}$ between the RIPC group and control group. The Produce Application and Support Software (PASS; Prentke Romich Company, Wooster, Ohio) was used to calculate the sample size.

Experiment 1: Effect of repeated limb RIPC on spinal cord $\mathbf{I} / \mathbf{R}$ injury. A total of 40 male Sprague-Dawley rats were randomly divided into 5 groups $(\mathrm{n}=8$ in each group):

1. Sham Group: Rats were operated on in the same way as in the other groups but without limb IPC and spinal cord ischemia.

2. Control group: After rats were anesthetized with $1 \%$ to $2 \%$ isoflurane for 30 minutes, spinal cord ischemia was induced.

3. RIPC2 MIN-3C GROUP: Three 2-minute cycles of right femoral artery occlusion and reperfusion were induced before spinal cord I/R.

4. RIPC3 MIN-3C GROUP: Three 3-minute cycles of right femoral artery occlusion and reperfusion were induced before spinal cord I/R.

5. RIPC5 MIN-3C GRouP: Three 5-minute cycles of right femoral artery occlusion and reperfusion were induced before spinal cord I/R. 
Thirty minutes after limb IPC (RIPC groups) or isoflurane inhalation (control group), the rats were subjected to spinal cord ischemia for $12 \mathrm{~min}$ utes except in the sham operation group.

Experiment 2: Effect of CB1 receptor or CB2 receptor antagonist on the ischemic tolerance induced by RIPC. Sixty-four male Sprague-Dawley rats were randomly divided into 8 groups ( $\mathrm{n}=8$ in each group):

1. G1 (CONTROL): The right femoral artery was isolated without occlusion, followed by spinal I/R protocol.

2. G2 (RIPC): Three 3-minute cycles of right femoral artery occlusion and reperfusion were induced before spinal cord I/R.

3. G3 (AM251 + RIPC): CB1 receptor antagonist AM251 (Tocris Bioscience, Bristol, United Kingdom; $1 \mathrm{mg} / \mathrm{kg}$ ) was intravenously administered 15 minutes before right femoral artery occlusion.

4. G4 (AM630+RIPC): CB2 receptor antagonist AM630 (Tocris; $1 \mathrm{mg} / \mathrm{kg}$ ) was intravenously administered 15 minutes before right femoral artery occlusion.

5. G5 (VEHICLE + RIPC): Vehicle was intravenously administered 15 minutes before right femoral artery occlusion (3 minutes) and reperfusion ( 3 minutes, 3 cycles) before spinal cord I/R protocol.

6. G6 (AM251): AM251 (Tocris; $1 \mathrm{mg} / \mathrm{kg}$ ) was intravenously administered 15 minutes before the sham operation of RIPC.

7. G7 (AM630): AM630 (Tocris;, $1 \mathrm{mg} / \mathrm{kg}$ ) was intravenously administered 15 minutes before the sham operation of RIPC.

8. G8 (VEHICLE): Vehicle was intravenously administered 15 minutes before the sham operation of RIPC.

Thirty minutes after preconditioning or 45 minutes after chemical pretreatment, the rats were subjected to spinal cord ischemia for 12 minutes. The time of total anesthesia was identical in the 8 groups.

Experiment 3. The contents of AEA and 2-AG in lumbar spinal cord samples before and after spinal cord ischemia. Twenty-four rats were divided into 2 groups: control group and RIPC group ( $\mathrm{n}=12$ in each group). Animals were humanely killed before (30 minutes after RIPC or sham operation) or 1 hour after spinal cord ischemia. The spinal cord was removed within 1 minute $\left(-20^{\circ} \mathrm{C}\right)$. A sample of 1 $\mathrm{cm}$ from L4 to L6 segments of spinal cord was frozen in Dry Ice and then stored at $-80^{\circ} \mathrm{C}$ in a refrigerator. For endocannabinoid measurement, spinal cord samples from the rats of control or RIPC groups $(n=6$ in each time point) were sampled.

\section{Endocannabinoid Measurement}

Endocannabinoids were extracted from the spinal cords. Animals were killed at specified time points. The spinal cord were removed and frozen on Dry Ice less than 1 minute after death. Tissue was stored at $-80^{\circ} \mathrm{C}$ until extraction. Tissue was homogenized with a glass rod and followed by incubation overnight at $-10{ }^{\circ} \mathrm{C}$ to precipitate proteins. A $0.2-\mathrm{mL}$ aliquot of each sample was transferred to a polypropylene tube. Then, $2 \mathrm{~mL}$ of ethyl acetate (liquid chromatography grade) was added to the tube and vortex mixed for 1 minute. After centrifugation (10 minutes at $3000 \mathrm{~g}$ ), the supernatant was transferred to a fresh tube. The deposition was then added to $2 \mathrm{~mL}$ ethyl acetate and operated in the same way. Next the supernatant was transferred to a new glass tube and evaporated under $\mathrm{N}_{2}$ gas. The final residue was dissolved in $50 \mu \mathrm{L}$ mobile phase with vortex mixing for 60 seconds. An aliquot of $10 \mu \mathrm{L}$ was subjected to the liquid chromatography/electrospray ionization tandem mass spectrometry.

AEA and 2-AG were separated on a $\mathrm{C}_{18}(50 \times 2.1 \mathrm{~mm}, 5 \mu \mathrm{m})$ reversephase column(Waters Symmetry; Waters Corporation, Milford, Mass). The mobile phase composition was a mixture of water/acetonitrile (10:90, vol/ vol) at a flow rate of $0.2 \mathrm{~mL} / \mathrm{min}$. AEA and 2-AG were separated on a reversed-phase column (Symmetry C18 column, $50 \times 2.1 \mathrm{~mm}, 5 \mu \mathrm{m}$ ) with an isocratic mobile phase consisting of $0.1 \%$ acetic acid water and acetonitrile (10:90, vol/vol). The column temperature and flow rate were $25^{\circ} \mathrm{C}$ and $0.2 \mathrm{~mL} / \mathrm{min}$, respectively. The mass spectrometer was operated using electrospray ionization with a capillary voltage of $+3500 \mathrm{~V}$. The positive ion multiple-reaction-monitoring mode analysis was performed using argon as the collision gas. Standard solutions of AEA and 2-AG were applied to optimize the detecting condition in the presence of liquid chromatography mobile phase. Data acquisition and processing were performed by MassLynx 4.1 software package (Waters Corporation).The AEA and 2-AG levels were normalized to the total weight of the wet tissue ( $\mathrm{pmol} / \mathrm{mg}$ tissue).

\section{Statistical Analysis}

Statistical analysis of physiologic data, number of normal neurons in the anterior cord, and level of endocannabinoids in the spinal cord were performed by one-way analysis of variance followed by a Student-Newman-Keuls test for multiple comparisons. For analysis of neurologic outcome in the individual occlusion group, significant overall values were obtained with a Kruskal-Wallis test. Specific comparisons between experimental groups were performed with the Mann-Whitney $U$ test for independent means. Data were expressed as mean \pm SEM.

\section{RESULTS}

\section{Experiment 1}

Physiologic variables. The hemodynamic variables are shown in Figure 1. Except for the sham group, the rectal temperature, arterial $\mathrm{pH}, \mathrm{PaCO}_{2}, \mathrm{PaO}_{2}$, and blood glucose concentrations were similar in all the groups. In groups in which spinal cord ischemia was induced, blood glucose was elevated and $\mathrm{pH}$ was decreased after reperfusion, but there was no difference among groups (data are not shown). Five minutes after reperfusion, the value of the distal blood pressure had recovered to nearly preischemic level (see Figure 1).

Neurologic outcome. All animals survived until the final neurologic behavior assessment at 48 hours after reperfusion. There was no neurologic change in the sham group, and all of the other groups of rats showed motor deficit in varying degrees. The neurologic outcome in the RIPC3 min-3c group was better than that of the control, RIPC2 min-3c, and RIPC5 min-3c groups, respectively $(P<.01)$. Three rats in the RIPC 3 min-3c group showed complete normal motor function (motor deficit index, MDI $=0$ ). The hind-limb MDIs of 5 groups at 48 hours after reperfusion are shown in Figure 2, A.

Histopathologic evaluation. The number of normal neurons in the RIPC 3 min-3c group was significantly greater than that in the RIPC2 min-3c, RIPC5 min-3c, and control groups $(P<.01)$. No difference was found in the number of normal neurons at the anterior spinal cord among the control, RIPC2 min-3c, and RIPC5 min-3c (Figure 2, $B$ ).

\section{Experiment 2}

Physiologic variables. The hemodynamics, rectal temperature, arterial $\mathrm{pH}, \mathrm{PaCO}_{2}, \mathrm{PaO}_{2}$, and blood glucose 

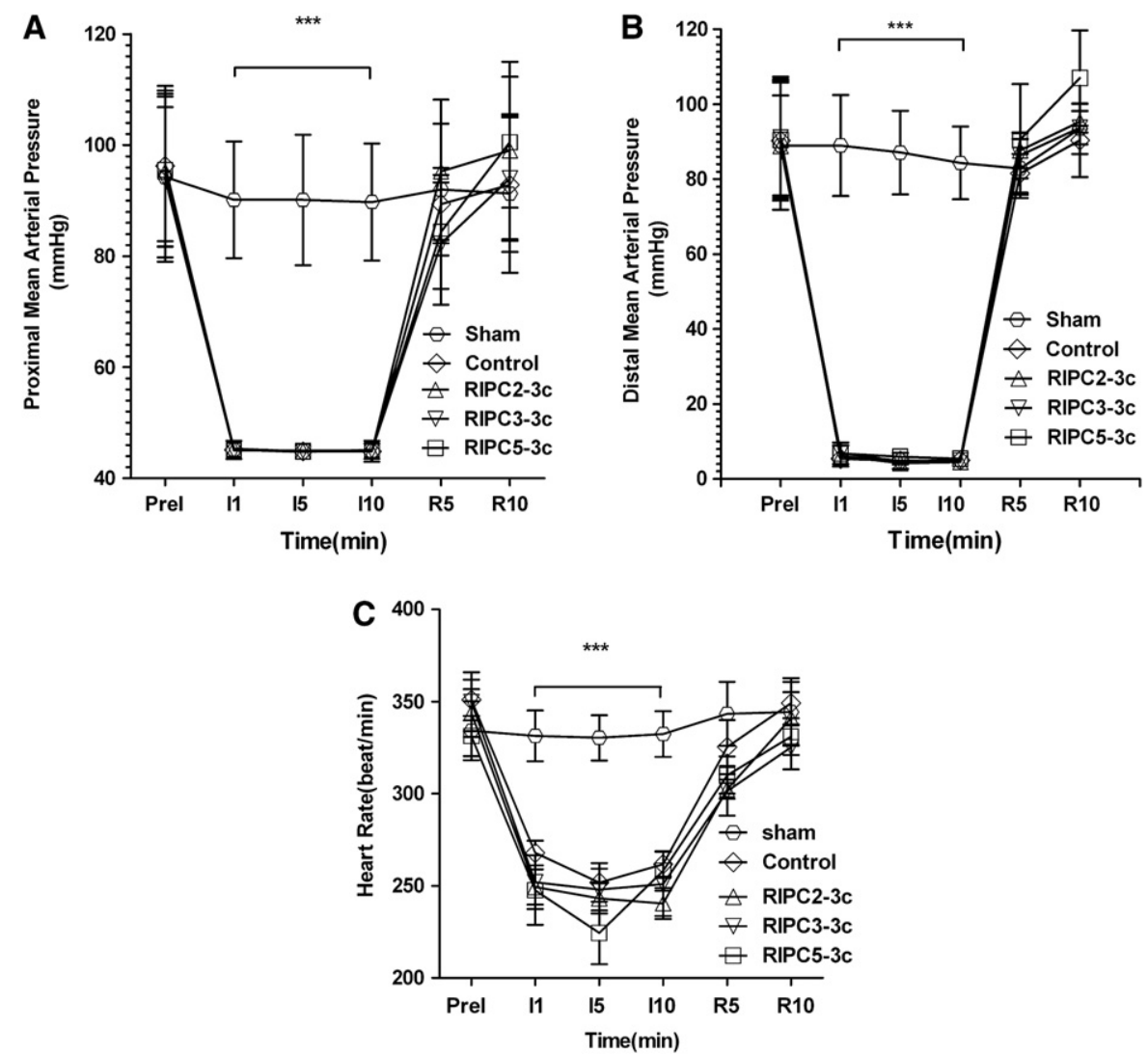

FIGURE 1. Proximal mean arterial pressure (A), distal mean arterial pressure (B), and heart rate (C) changes during surgical procedure in experiment 1. Sham, Sham operation without spinal cord ischemia; Control, sham operation of remote preconditioning before spinal cord ischemia; $R I P C 2-3 c$, rats accepted remote ischemic preconditioning ( 3 cycles, 2 minutes of ischemia/2 minutes of reperfusion) before spinal cord ischemia; RIPC 3-3c, rats accepted remote ischemic preconditioning ( 3 cycles, 3 minutes of ischemia/ 3 minutes of reperfusion) before spinal cord ischemia; RIPC 5 -3c, rats accepted remote ischemic preconditioning ( 3 cycles, 5 minutes of ischemia/5 minutes of reperfusion) before spinal cord ischemia. $I$, Ischemia; $R$, reperfusion. The data are expressed as mean \pm SEM. $* * * P<.001$ vs sham.

concentration changes were similar to those in experiment 1 (data not shown).

Neurologic outcome. All animals survived until the final neurologic behavior assessment at 48 hours after reperfusion. The neurologic outcome in the RIPC group was better than that of the control $(P<.05)$ and AM251+RIPC groups $(P<.01)$. The MDI in RIPC, AM630+ RIPC, and vehicle+ RIPC3 groups did not show significant differences. There was no significant difference among control, AM251 + RIPC, AM251, AM630, and vehicle groups. The hindlimb MDIs at 48 hours after reperfusion are shown in Figure 3, $A$.

Histopathologic evaluation. The number of normal motor neurons in the RIPC group was significantly more than that in the control $(P<.05)$ and AM251 + RIPC groups $(P<$ $.05)$. There was no significance among RIPC, AM630 + RIPC, and vehicle + RIPC groups. The ventral neurons in AM251, AM630, and vehicle groups were damaged seriously, and the number of normal neurons among these groups did not show significant differences (Figure 3,B).

\section{Experiment 3}

Levels of AEA and 2-AG in lumbar spinal cord before and after spinal cord ischemia in the RIPC and control groups are shown in Figure 4. The preischemic AEA level in the RIPC group was higher than that in the control group $(P<.05)$. The results indicated that RIPC ( 3 cycles of 3 minutes of limb I/R) stimulated the formation of AEA in the lumbar spinal cord. The postischemic AEA level did not show significant difference between the 2 groups. There was no significant difference in 2-AG content in lumber spinal cord at corresponding times between the 2 groups.

\section{DISCUSSION}

This study provided evidence that the endogenous CB system contributes to the neuroprotective effects of limb RIPC on spinal cord ischemia, conferred by a brief episode of femoral artery occlusion and reperfusion. Our study also proved that 3 cycles of 3 minutes of ischemia and 3 minutes of reperfusion provided the optimal conditions of limb RIPC on the induction of spinal cord ischemic tolerance in the rat 

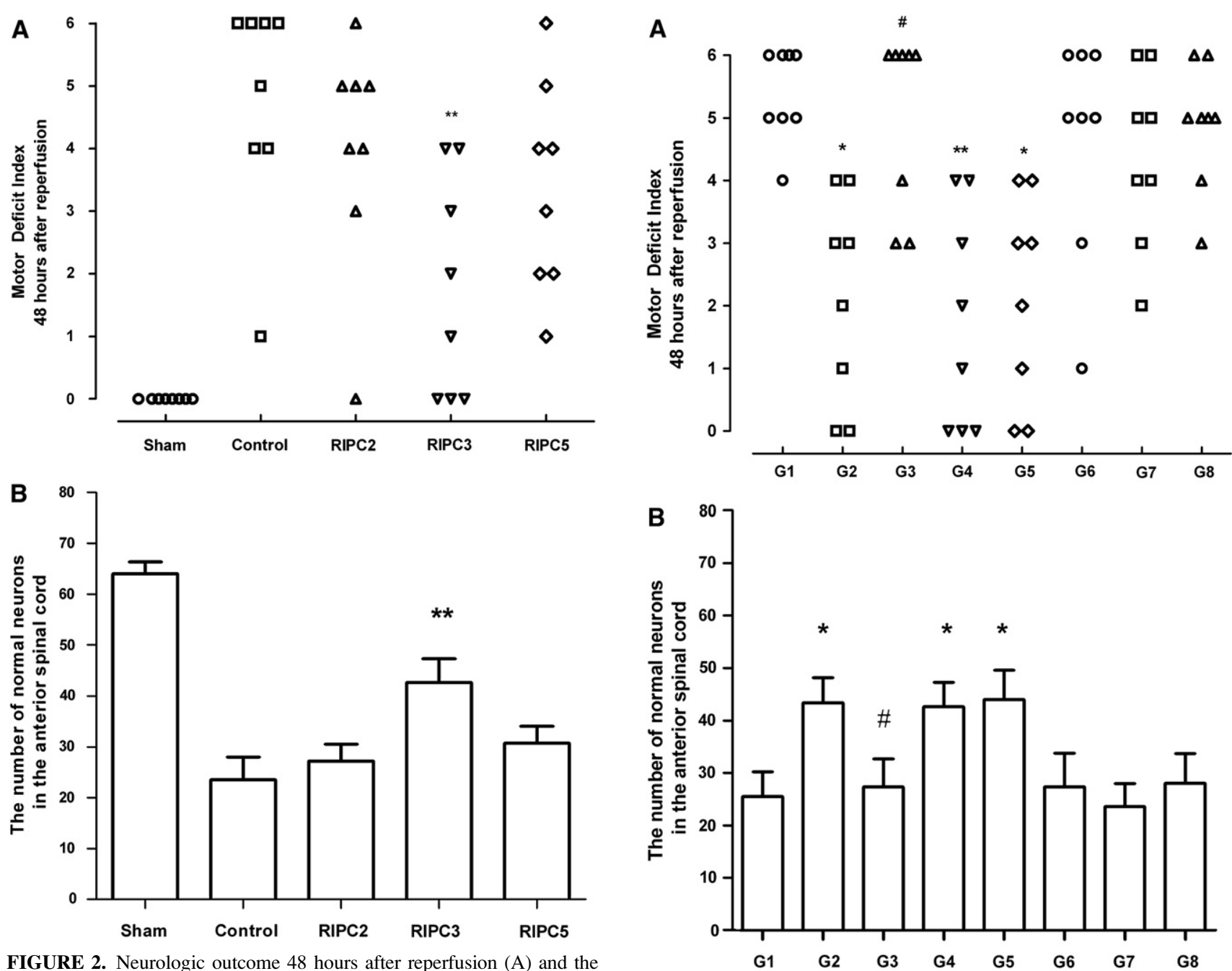

FIGURE 2. Neurologic outcome 48 hours after reperfusion (A) and the number of normal neurons in the anterior spinal cord in experiment 1 (B). (**P $<0.01$ vs. control). Sham, Sham operation without spinal cord ischemia; Control, sham operation of remote preconditioning before spinal cord ischemia; RIPC2-3c, rats accepted remote ischemic preconditioning ( 3 cycles, 2 minutes of ischemia/2 minutes of reperfusion) before spinal cord ischemia; RIPC $3-3 c$, rats accepted remote ischemic preconditioning ( 3 cycles, 3 minutes of ischemia/ 3 minutes of reperfusion) before spinal cord ischemia; RIPC 5-3c, rats accepted remote ischemic preconditioning (3 cycles, 5 minutes of ischemia/5 minutes of reperfusion) before spinal cord ischemia.

model. Limb RIPC elevated the AEA content in the spinal cord. Furthermore, the neuroprotective effects of limb RIPC were reversed by pretreatment with $\mathrm{CB} 1$ receptor antagonist. These observations suggested that spinal cord ischemic tolerance induced by limb RIPC was mediated through CB1 receptors.

Spinal cord injury after a successful surgical operation on the thoracic aorta is an unpredictable but disastrous complication. The causes of acute spinal cord dysfunction were believed to be the result of a spinal cord I/R injury during aortic crossclamping. Excitatory amino acids, heat shock protein,

FIGURE 3. Neurologic outcome 48 hours after reperfusion (A) and the number of normal neurons in the anterior spinal cord in experiment 2 (B). $* P<.05$ vs G1; $* * P<0.01$ vs G1; \#P<.05 vs G2. G1, Control group; $G 2$, rats accepted remote ischemic preconditioning ( 3 cycles, 3 minutes of ischemia/3 minutes of reperfusion) before spinal cord ischemia; G3, AM251 was intravenously administered 15 minutes before remote preconditioning; $G 4$, AM630 was intravenously administered 15 minutes before remote preconditioning; G5, vehicle was intravenously administered 15 minutes before remote preconditioning; G6, AM251 was intravenously administered 15 minutes before sham operation of remote preconditioning; $G 7$, AM630 was intravenously administered 15 minutes before sham operation of remote preconditioning; $G 8$, vehicle was intravenously administered 15 minutes before sham operation of remote preconditioning.

and free radicals were all suggested to play important roles in the development of spinal cord ischemic injury. However, ameliorative measures, including hypothermia and free radical scavengers, were still inadequate. IPC has been shown to provide spinal cord protection from ischemic injury. ${ }^{15,16}$ However, exposure of the spinal cord to a brief period of ischemia before an anticipated ischemic event did not appear to be clinically relevant. RIPC was a protective phenomenon 

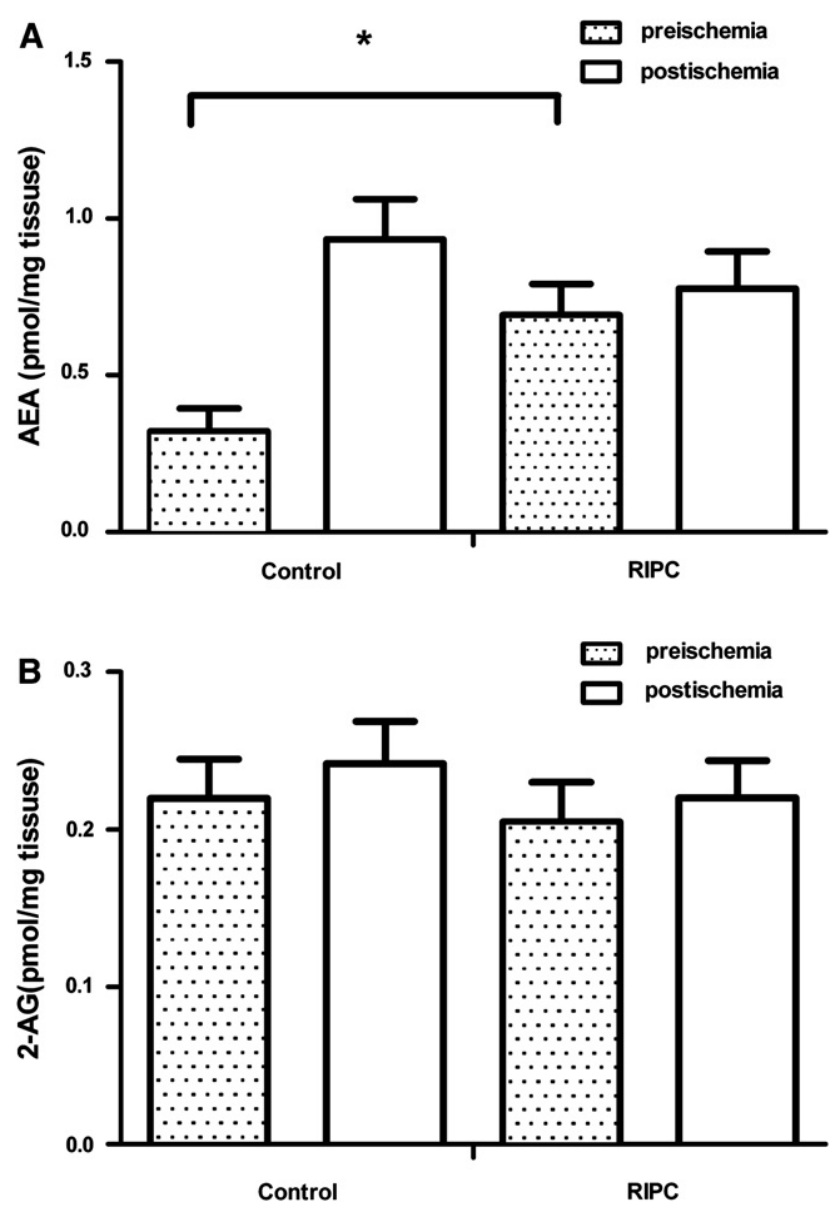

FIGURE 4. Levels of arachidonylethanolamide ( $A E A$ ) (A) and 2-arachidonoylglycerol $(2-A G)(\mathrm{B})$ in lumbar spinal cord at preischemia (30 minutes after limb remote preconditioning [RIPC] or sham operation) and postischemia (1 hour after spinal cord ischemia) derived from RIPC and control groups. Data are expressed as mean $\pm \mathrm{SEM} ; \mathrm{n}=6$ per group. $* P<.05$ compared with controls. Control group, Sham operation of remote preconditioning was performed before spinal cord ischemia; RIPC group, limb remote preconditioning was induced before spinal cord ischemia.

first described by Przyklenk and associates. ${ }^{17} \mathrm{~A}$ recent study has demonstrated that RIPC of the kidney can induce ischemic tolerance in the spinal cord in a rabbit model. ${ }^{18}$ However, inducing a brief period of kidney ischemia before an anticipated spinal cord ischemic event seemed unsafe and not practicable.

The current study confirmed that 3 cycles of brief limb I/R episodes (RIPC) applied before aortic occlusion result in significant protection of the spinal cord from ischemic injury in a rat model, although neurologic function in the RIPC 3 min$3 \mathrm{c}$ group was better than that in the control group. The number of normal motor neurons in the ventral horn in the RIPC3 min- $3 c$ and RIPC5 min-3c groups was higher than that in the control group. Inasmuch as it was easier and safer to perform a limb RIPC than to induce brief ischemia on the aorta itself before the thoracic aorta surgery, our finding has significant clinical applications in the future. The spinal cord ischemia model that was used in the present study is referred to in previous reports. ${ }^{13}$ This model was a reliable and stable animal model for studying neuroprotective manipulations and mechanisms on spinal cord in rodents.

The endocannabinoid system represented a pivotal neuroprotective mechanism in ischemic and traumatic neuronal injuries. Modulation of immune responses, activation of cytoprotective signaling pathways, and antioxidant properties of CBs have been suggested to be involved in the mechanisms of the neuroprotective effects of the endocannabinoid system. ${ }^{19}$ Previous study demonstrated that CB receptor agonist attenuated hippocampal neuronal loss after cerebral ischemia in a CB1-dependent manner. ${ }^{20}$ Knockout of CB1 elevated mortality and increased infarct size from cerebral ischemia in mice. ${ }^{21}$ A protective effect on spinal cord neurons was also induced by the pharmacologic modulation of the endocannabinoid system. ${ }^{22}$ However, the role of endocannabinoids in spinal cord ischemia and RIPC-induced ischemic tolerance remained to be elucidated.

Several studies have proved that an increase of endocannabinoid levels was found after cerebral and myocardial $\mathrm{I} / \mathrm{R}$ injury. ${ }^{23,24}$ It was speculated that the increase of endocannabinoid levels in tissues may related to the cell damage. Anandamide (AEA) and 2-AG are the natural ligands of $\mathrm{CB}$ receptor that have been extensively studied so far. Nucci and associates ${ }^{25}$ found that the I/R-induced cell death was attenuated by the AEA stable analog methanandamide, suggesting that endogenous AEA may play a protective role against $\mathrm{I} / \mathrm{R}$ injury. ${ }^{25} \mathrm{~A}$ recent study demonstrated that endogenous $\mathrm{CBs}$, through acting on $\mathrm{CB}_{2}$ receptors, are involved in the cardioprotective phenomenon of RIPC. ${ }^{12}$ This study suggested that elevating the endogenous CB level before a lethal ischemia can protect the fetal organ from cell death by acting on $\mathrm{CB}$ receptors. In the current study, we found that AEA, rather than 2-AG, was increased at the early phase after RIPC and spinal cord ischemia. This was consistent with most recent studies, which have found that spinal cord lesions induced an increase of AEA levels in early stages and an increase in 2-AG level in late stages. ${ }^{26}$ The elevation of AEA may be attributed to the ischemic tolerance on spinal cord induced by RIPC.

It has been proved that $\mathrm{I} / \mathrm{R}$, but not ischemia alone, triggers increases in the levels of the endocannabinoids. ${ }^{27}$ Furthermore, these increases were positively correlated with the degree of serum tumor necrosis factor- $\alpha$, macrophage inflammatory protein- $1 \alpha$, and macrophage inflammatory protein-2 levels. Consistently, brief exposure of cells to various oxidants (hydrogen peroxide, peroxynitrite) or inflammatory stimuli (tumor necrosis factor- $\alpha)^{28}$ triggered marked increases in cellular endocannabinoids levels. These findings suggest that a variety of triggers, such as reactive oxygen species, inflammatory cytokines, and others, may induce the elevation of endocannabinoids. In previous studies by 
us $^{29}$ and by Shahid and associates, ${ }^{30}$ an increase of reactive oxygen species in the serum and spinal cord was found after limb RIPC. ${ }^{29,30}$ Thus we speculated that the first possibility of elevation of endocannabinoid content in spinal cord might be triggered by circulating reactive oxygen species induced by limb ischemia. In a recent study, Opitz and coworkers ${ }^{31}$ found that stimulation with the proinflammatory cytokine increased the release endocannabinoids by endothelial progenitor cells. Inasmuch as limb ischemia causes the increase of inflammatory factors, the second possibility is that elevation of endocannabinoid content might be caused by endothelial progenitor cell- released endocannabinoids after limb ischemia. As mentioned above, both reactive oxygen species and inflammatory factors are circulating mediators and can activate the release of endocannabinoids directly or indirectly (through the endothelial progenitor cells) after limb RIPC.

Two CB receptors have been identified, CB1 and CB2 receptors. AM251 was used as a specific $\mathrm{CB} 1$ receptor antagonist and AM630 as a specific $\mathrm{CB} 2$ receptor antagonist in the current study. The dosages of AM251 and AM630 were referred to previous study. ${ }^{12}$ The results showed that neuroprotective effects induced by limb RIPC were reversed by pretreatment of AM251 rather than AM630. These findings indicated that $\mathrm{CB} 1$ receptor plays a crucial role in inducing ischemic tolerance of RIPC in spinal cord.

The exact mechanisms of CB1 receptor on the neuroprotective effect are still unclear. Farquhar-Smith and associates $^{32}$ found that CB1 receptors distribute mainly in the dorsolateral funiculus, the superficial dorsal horn, and lamina $\mathrm{X}$ in lumber spinal cords, which is the sensor neuron area. Nakayama and coworkers ${ }^{33}$ demonstrated that atrial natriuretic peptide reduces $\mathrm{I} / \mathrm{R}$-induced spinal cord injury by enhancing sensory neuron activation in vivo. On activation, sensory neurons release calcitonin gene-related peptide, thereby increasing endothelial production of prostaglandin $I_{2}$, which contributes to reduction of spinal cord injury in rats subjected to spinal cord $\mathrm{I} / \mathrm{R}$ by inhibiting inflammatory response. In the present study, CB1 receptor antagonist has reversed the protective effect of RIPC, which implies the protective effect is mediated by CB1 receptor. The study by Fioravanti and colleagues ${ }^{34}$ showed that constitutive activity at the CB1 receptor maintains the transient receptor potential vanilloid 1 channel in a sensitized state responsive to noxious chemical stimuli. Considering the previous reports with our findings in the current study, we speculated that RIPC induces an increase of endocannabinoids in the spinal cord, the latter bind to CB1 receptor, which activate transient receptor potential vanilloid 1 channel and induce calcitonin gene-related peptide release. The process elevated the level of prostaglandin $I_{2}$ in the spinal cord, which induces spinal cord ischemic tolerance in the rat.

In the current study, we only investigated the RIPC effect at 24 and 48 hours after reperfusion, which is a relatively short period of time, to demonstrate the manifestation of delayed neuronal necrosis from apoptotic mechanisms, or other mechanisms related to delayed neuronal necrosis. Meanwhile, the exact mechanisms of endocannabinoid elevation in the spinal cord after limb ischemia (RIPC) were not clarified by this study. We speculated that both reactive oxygen species and endothelial progenitor cell mechanisms are involved in the elevation of endocannabinoids in the spinal cord after RIPC. All of these need to be elucidated in a future study.

In summary, the findings of this study provide evidence of the contribution of endogenous cannabinoids to neuroprotection induced by a brief episode of femoral artery occlusion and reperfusion. These neuroprotective effects of endogenous cannabinoids on $\mathrm{I} / \mathrm{R}$-induced injury in spinal cord are mediated by CB1 receptors.

\section{References}

1. Svensson LG, Crawford ES, Hess KR, Coselli JS, Safi HJ. Experience with 1509 patients undergoing thoracoabdominal aortic operations. J Vasc Surg. 1993;17: 357-68.

2. Yamazaki M, Mochizuki M, Ikeda Y, Sodeyama T, Okawa A, Koda M, et al. Clinical results of surgery for thoracic myelopathy caused by ossification of the posterior longitudinal ligament: operative indication of posterior decompression with instrumented fusion. Spine. 2006;31:1452-60.

3. Abraham VS, Swain JA, Forgash AJ, Williams BL, Musulin MM. Ischemic preconditioning protects against paraplegia after transient aortic occlusion in the rat Ann Thorac Surg. 2000;69:475-9.

4. Toumpoulis IK, Anagnostopoulos CE, Drossos GE, Malamou-Mitsi VD, Pappa LS, Katritsis DG. Early ischemic preconditioning without hypotension prevents spinal cord injury caused by descending thoracic aortic occlusion. J Thorac Cardiovasc Surg. 2003;125:1030-6.

5. Dong H, Xiong L, Zhu Z, Chen S, Hou L, Sakabe T. Preconditioning with hyperbaric oxygen and hyperoxia induces tolerance against spinal cord ischemia in rabbits. Anesthesiology. 2002;96:907-12.

6. Nie H, Xiong L, Lao N, Chen S, Xu N, Zhu Z. Hyperbaric oxygen preconditioning induces tolerance against spinal cord ischemia by upregulation of antioxidant enzymes in rabbits. J Cereb Blood Flow Metab. 2006;26:666-74.

7. Sang H, Cao L, Qiu P, Xiong L, Wang R, Yan G. Isoflurane produces delayed preconditioning against spinal cord ischemic injury via release of free radicals in rabbits. Anesthesiology. 2006;105:953-60.

8. Walsh SR, Tang T, Sadat U, Dutka DP, Gaunt ME. Cardioprotection by remote ischaemic preconditioning. Br J Anaesth. 2007;99:611-6.

9. Loukogeorgakis SP, Panagiotidou AT, Broadhead MW, Donald A, Deanfield JE MacAllister RJ. Remote ischemic preconditioning provides early and late protection against endothelial ischemia-reperfusion injury in humans: role of the autonomic nervous system. J Am Coll Cardiol. 2005;46:450-6.

10. Hausenloy DJ, Mwamure PK, Venugopal V, Harris J, Barnard M, Grundy E, et al Effect of remote ischaemic preconditioning on myocardial injury in patients undergoing coronary artery bypass graft surgery: a randomised controlled trial. Lancet. 2007;370:575-9.

11. Pacher P, Batkai S, Kunos G. The endocannabinoid system as an emerging target of pharmacotherapy. Pharmacol Rev. 2006;58:389-462.

12. Hajrasouliha AR, Tavakoli S, Ghasemi M, Jabehdar-Maralani P, Sadeghipour H, Ebrahimi F, et al. Endogenous cannabinoids contribute to remote ischemic preconditioning via cannabinoid CB2 receptors in the rat heart. Eur J Pharmacol. 2008;579:246-52.

13. Taira Y, Marsala M. Effect of proximal arterial perfusion pressure on function, spinal cord blood flow, and histopathologic changes after increasing intervals of aortic occlusion in the rat. Stroke. 1996;27:1850-8.

14. Cizkova D, Carmel JB, Yamamoto K, Kakinohana O, Sun D, Hart RP, et al. Characterization of spinal HSP72 induction and development of ischemic tolerance after spinal ischemia in rats. Exp Neurol. 2004;185:97-108.

15. Wan IY, Angelini GD, Bryan AJ, Ryder I, Underwood MJ. Prevention of spinal cord ischaemia during descending thoracic and thoracoabdominal aortic surgery. Eur J Cardiothorac Surg. 2001;19:203-13. 
16. Kakimoto M, Kawaguchi M, Sakamoto T, Inoue S, Furuya H, Nakamura M, et al. Evaluation of rapid ischemic preconditioning in a rabbit model of spinal cord ischemia. Anesthesiology. 2003;99:1112-7.

17. Przyklenk K, Bauer B, Ovize M, Kloner RA, Whittaker P. Regional ischemic "preconditioning" protects remote virgin myocardium from subsequent sustained coronary occlusion. Circulation. 1993;87:893-9.

18. Gurcun U, Discigil B, Boga M, Ozkisacik E, Badak MI, Yenisey C, et al. Is remote preconditioning as effective as direct ischemic preconditioning in preventing spinal cord ischemic injury? J Surg Res. 2006;135:385-93.

19. Pacher P, Hasko G. Endocannabinoids and cannabinoid receptors in ischaemiareperfusion injury and preconditioning. Br J Pharmacol. 2008;153:252-62.

20. Nagayama T, Sinor AD, Simon RP, Chen J, Graham SH, Jin K, et al. Cannabinoids and neuroprotection in global and focal cerebral ischemia and in neuronal cultures. J Neurosci. 1999;19:2987-95.

21. Parmentier-Batteur S, Jin K, Mao XO, Xie L, Greenberg DA. Increased severity of stroke in CB1 cannabinoid receptor knock-out mice. J Neurosci. 2002;22:9771-5.

22. Abood ME, Rizvi G, Sallapudi N, McAllister SD. Activation of the CB1 cannabinoid receptor protects cultured mouse spinal neurons against excitotoxicity. Neurosci Lett. 2001;309:197-201.

23. Muthian S, Rademacher DJ, Roelke CT, Gross GJ, Hillard CJ. Anandamide content is increased and CB1 cannabinoid receptor blockade is protective during transient, focal cerebral ischemia. Neuroscience. 2004;129:743-50.

24. Wagner JA, Hu K, Bauersachs J, Karcher J, Wiesler M, Goparaju SK, et al. Endogenous cannabinoids mediate hypotension after experimental myocardial infarction. J Am Coll Cardiol. 2001;38:2048-54.

25. Nucci C, Gasperi V, Tartaglione R, Cerulli A, Terrinoni A, Bari M, et al. Involvement of the endocannabinoid system in retinal damage after high intraocular pressure-induced ischemia in rats. Invest Ophthalmol Vis Sci. 2007;48:2997-3004.
26. Garcia-Ovejero D, Revalo-Martin A, Petrosino S, Docagne F, Hagen C, Bisogno T, et al. The endocannabinoid system is modulated in response to spinal cord injury in rats. Neurobiol Dis. 2009;33:57-71.

27. Batkai S, Osei-Hyiaman D, Pan H, El-Assal O, Rajesh M, Mukhopadhyay P, et al. Cannabinoid-2 receptor mediates protection against hepatic ischemia/reperfusion injury. FASEB J. 2007;21:1788-800.

28. Pacher P, Beckman JS, Liaudet L. Nitric oxide and peroxynitrite in health and disease. Physiol Rev. 2007;87:315-424.

29. Zhang Y, Xiong L, Dong H. Limb remote ischemic preconditioning induces tolerance against spinal cord ischemia by upregulation of antioxidant enzymes activities in rabbits. Prog Mod Biomed (Chinese). 2007;7:1127-30.

30. Shahid M, Tauseef M, Sharma KK, Fahim M. Brief femoral artery ischaemia provides protection against myocardial ischaemia-reperfusion injury in rats: the possible mechanisms. Exp Physiol. 2008;93:954-68.

31. Opitz CA, Rimmerman N, Zhang Y, Mead LE, Yoder MC, Ingram DA, et al. Production of the endocannabinoids anandamide and 2-arachidonoylglycerol by endothelial progenitor cells. FEBS Lett. 2007;581:4927-31.

32. Farquhar-Smith WP, Egertova M, Bradbury EJ, McMahon SB, Rice AS, Elphick MR. Cannabinoid $\mathrm{CB}(1)$ receptor expression in rat spinal cord. $\mathrm{Mol}$ Cell Neurosci. 2000;15:510-21.

33. Nakayama T, Harada N, Asano M, Nomura N, Saito T, Mishima A, et al. Atrial natriuretic peptide reduces ischemia/reperfusion-induced spinal cord injury in rats by enhancing sensory neuron activation. J Pharmacol Exp Ther. 2007;322: 582-90.

34. Fioravanti B, De Felice M, Stucky CL, Medler KA, Luo MC, Gardell LR, et al. Constitutive activity at the cannabinoid $\mathrm{CB} 1$ receptor is required for behavioral response to noxious chemical stimulation of TRPV1: antinociceptive actions of CB1 inverse agonists. J Neurosci. 2008;28:11593-602. 